\title{
Immunocytochemical localization of glucose 6-phosphatase and cytosolic phosphoenolpyruvate carboxykinase in gluconeogenic tissues reveals unsuspected metabolic zonation
}

Fabienne Rajas · Hélène Jourdan-Pineau •

Anne Stefanutti • Elham Abou Mrad •

Patrick B. Iynedjian • Gilles Mithieux

Published online: 30 January 2007

(C) Springer-Verlag 2007

\section{Erratum to: Histochem Cell Biol DOI 10.1007/s00418-006-0263-5}

The name of the fourth author, Elham Abou Mrad, was rendered in the affiliations incorrectly as E. A. Mrad. It should be E. Abou Mrad.
The first affiliation (F. Rajas, H. Jourdan-Pineau, A. Stefanutti, G. Mithieux) should correctly read:

Inserm, U855, Lyon, F-69008, France

The online version of the original article can be found at http://dx.doi.org/10.1007/s00418-006-0263-5.

F. Rajas · H. Jourdan-Pineau · A. Stefanutti ·

G. Mithieux

Inserm, U855, Lyon, F-69008, France

F. Rajas · H. Jourdan-Pineau - A. Stefanutti ·

E. Abou Mrad · G. Mithieux

université de Lyon, Lyon, F-69008, France

F. Rajas · H. Jourdan-Pineau · A. Stefanutti ·

E. Abou Mrad · G. Mithieux

université Lyon 1, Lyon, F-69008, France

F. Rajas · H. Jourdan-Pineau · A. Stefanutti ·

E. Abou Mrad - G. Mithieux

INSERM, IFR62, Lyon, F-69008, France

P. B. Iynedjian

Department of Cell Physiology and Metabolism,

University of Geneva School of Medicine,

1211 Geneva, Switzerland

F. Rajas $(\bowtie)$

INSERM U855, Faculté de Médecine Laennec,

Rue Guillaume Paradin, 69372 Lyon cedex 08, France

e-mail: rajas@sante.univ-lyon1.fr

E. Abou Mrad

Inserm U870, Lyon, F-69008, France 